\title{
Is there a relationship between the morphological characteristics of developmental venous anomalies and the presence of parenchymal abnormalities?
}

\author{
Ayşe Nur Şirin Özcan ${ }^{1}$, Behruz Hasanov²®, Özlem Ünal \\ ${ }^{1}$ Department of Radiology, University of Health Sciences, Ankara Bilkent City Hospital, Ankara, Turkey \\ ${ }^{2}$ Department of Radiology, Ankara Yıldırım Beyazit University School of Medicine, Ankara, Turkey
}

\begin{abstract}
Objectives: Developmental venous anomaly (DVA) is a benign vascular malformation. Hyperintensity in the fluid-attenuated inversion recovery (FLAIR) sequence in the adjacent white matter has been previously identified. Although there are different theories, it is not yet clear why these hyperintensities occur. In this study, we aimed to investigate the factors affecting hyperintensity formation by examining the relationship between the morphological findings of DVA and the presence of FLAIR hyperintensity.

Methods: The study included 84 cases diagnosed with DVA. Patients with susceptibility weighted imaging (SWI) and/or postcontrast T1-weighted sequences in addition to conventional MRI sequences were included. The patients were divided into two groups as hyperintensity FLAIR (+) and FLAIR (-) in the examination performed around DVA. There were 24 patients in the FLAIR $(+)$ group and 60 patients in the FLAIR (-) group. The localization of DVAs, drainage localization of the intramedullary vein, collector vein diameter, collector vein length, DVA location depth, and patient ages were compared between the two groups.

Results: When examining the relationship between the groups and variables in the statistical evaluation, there was no difference between the groups in terms of DVA localization, the venous system of drainage, DVA depth, and the length of the collector vein. However, a significant difference was observed between the groups in terms of the collector vein diameter and age.

Conclusions: The results of this study indicating a relationship between collector vein diameter and patient age. The relationship between this diameter and age suggests that there is a correlation between the degree and duration of venous congestion and the change in white matter.
\end{abstract}

Keywords: Developmental venous anomaly, susceptibility weighted imaging, FLAIR hyperintensity

D evelopmental venous anomaly (DVA), also called venous angioma, is the most common vascular malformation in the brain [1]. Recently, it has become controversial concerning whether DVA is a vascular malformation or venous variation [1]. DVAs consist of a large number of small intramedullary veins that form the appearance of an umbrella or caput medusa, draining into a single collector vein. They are often considered benign and asymptomatic and are detected incidentally in imaging tests. There is no consensus on the formation mechanisms of DVAs; however, there are theories indicating that they

How to cite this article: Şirin Özcan AN, Hasanov B, Ünal Ö. Is there a relationship between the morphological characteristics of developmental venous anomalies and the presence of parenchymal abnormalities? Eur Res J 2020;7(3):256-264. DOI: 10.18621/eurj.734215

Address for correspondence: Ayşe Nur Şirin Özcan, MD., University of Health Sciences, Ankara Bilkent City Hospital, Department of Radiology, Bilkent/Lodumlu 06800,Ankara,Turkey.E-mail: aysenursirinozcan@gmail.com 
develop to compensate for an undeveloped cerebral venous system in a certain area of the brain parenchyma [2-4].

Although they are often asymptomatic, DVAs can also be symptomatic, causing venous infarction with the thrombosis of the collector vein. In addition, DVAs may be accompanied by cavernoma, white matter change in drainage localization, dystrophic calcification, and local atrophy [5-7].

DVAs are easily detected by susceptibility weighted imaging (SWI) or contrast-enhanced MRI and CT examinations. DVAs can be identified in conventional MRI sequences and CT if only they are large enough. During imaging, the large intramedullary vein and venous vascular structures draining into this vein are contrast-enhanced. Deoxygenated hemoglobin in the lumen of venous structures described in SWI sequences is used as an intrinsic contrast agent, allowing a kind of venography examination to be performed without using contrast media [8].

DVAs drain a certain parenchymal area, such as other cerebral venous structures. The intensity difference in white matter drained by DVA has been previously described [5-9]. Although white matter change is considered to occur due to venous hypertension, its pathophysiology remains unclear [10].

In this study, we aimed to investigate the factors affecting the formation of hyperintensity by examining the relationship between the morphological findings of DVAs and the presence or absence of FLAIR hyperintensity.

\section{METHODS}

This retrospective study was approved by the Bilkent City Hospital Ethics committee (E-19-108). The study was designed according to declaration of Helsinki and written consent was obtained from all participants.

The reports of brain MRI scans performed at the radiology department of our hospital between January 2017 and February 2019 were retrospectively screened. A total of 189 patients diagnosed with DVAs based on these reports were included in the study. In addition to the conventional sequences, the presence of contrast-enhanced examination and/or SWI sequence was accepted as a criterion for the diagnosis of DVA. Diffuse white matter disease, brain MRI scans not including SWI/post-contrast-enhanced T1weighted sequences were the exclusion criteria of the study.

Patients with white matter disease, which causes diffuse hyperintensity in white matter, was excluded from the study because it was not possible to determine whether hyperintensity formation was secondary to venous angioma or not. Two DVAs located in the brainstem were excluded due to the brain MRI scans not including SWI/post-contrast-enhanced T1weighted sequences.

A 3 Tesla system (SKYRA Siemens) with a 16channel head coil was used for the MRI examination. Conventional sequences consisted of axial T1weighted, axial T2-weighted, axial FLAIR, sagittal T2-weighted and axial diffusion examinations. Conventional sequences were accompanied by T1-weighted or SWI examination in two post-contrast-enhanced planes (axial and sagittal). For contrast enhancement, $0.1 \mathrm{mmol} / \mathrm{kg}$ gadolinium was used. The SWI examination consisted of magnitude, phase, and minimum intensity projection (MIP). The visualization of a DVA in one of the contrast-enhanced or SWI sequences was considered to be sufficient for the diagnosis.

The images of cases diagnosed based on brain MRI reports were reevaluated by a neuroradiologist with 13 years of experience. The diagnosis of DVA was made by demonstrating the drainage of thin venous segments into the wide collector vein, resulting in the typical caput medusa appearance in the contrast-enhanced T1-weighted or SWI sequence. For all DVA lesions, localization, drainage localization of the intramedullary vein, collector vein length, intramedullary vein diameter, FLAIR hyperintensity in the drainage area, and diffuse white matter hyperintensity extending to the drainage area were noted.

According to whether there was white matter hyperintensity around DVAs, the patients were divided into two groups as FLAIR $(+)$ and FLAIR (). White matter hyperintensity was evaluated using the axial FLAIR sequence due to its superiority to the T2weighted sequence in showing white matter lesions. Since the flow in the DVA lumen may also create hyperintensity in the FLAIR sequence, care was taken to ensure that hyperintensity was not at the lumen level when performing the evaluation. DVA-associ- 


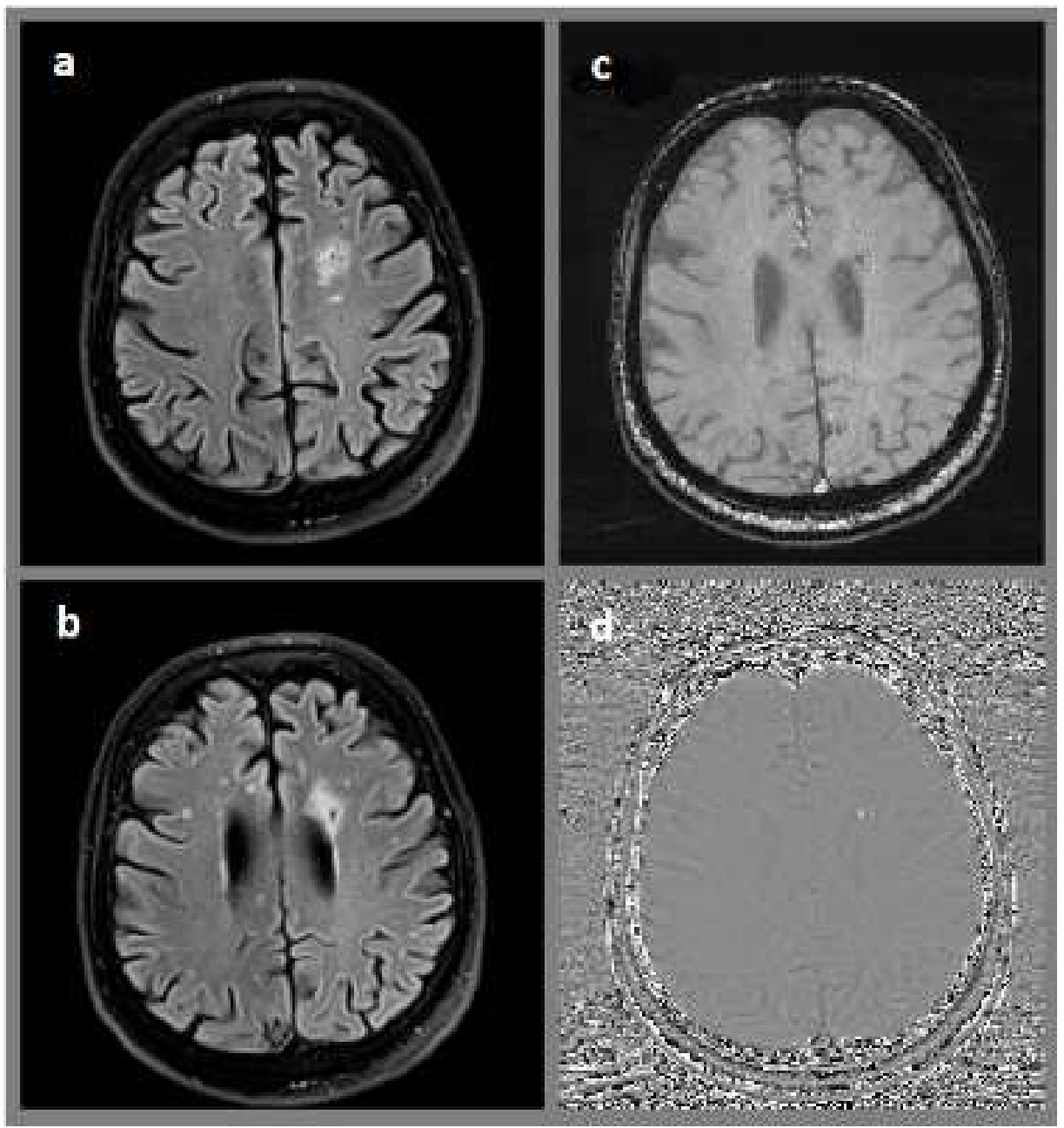

Fig. 1. (a, b) DVA associated hyperintensity at FLAIR sequence, (c, d) DVA collector vein at SWI and phase images.

ated signal abnormalities and DVA collector vein at SWI-Phase Images are shown in Fig. 1.

The lesions diagnosed with DVA based on the contrast-enhanced T1-weighted or SWI sequence were classified for both groups according to their following characteristics:

- Localization: DVAs located in cerebral lobes were defined as lobar, and basal ganglion, brainstem and cerebellum locations were classified separately.

- Depth: For this classification, the widely adopted method from Lee et al. [11] was used based on the localization of multiple small intramedullary veins draining into the collector vein. Accordingly, the DVAs were classified as periventricular, juxtacortical, and subcortical.

- Drainage system: Deep or superficial descriptions were used to indicate the venous system into the vein drained. DVAs that equally participated in both systems were noted as D+Y.
- Length of the collector vein: DVAs were visually classified as small, medium, and large as previously described by Santucci et al. [9]

- Width: This classification was made visually, taking into account the size of the intramedullary vein in the contrast-enhanced T1-weighted or SWI sequence. DVAs were classified as 'thin', 'medium', and 'wide'. Of the DVAs that were not seen in conventional sequences, those that were visualized in SWI or contrast-enhanced T1-weighted sequence were classified as 'thin' if they appeared as a thin line, 'wide' if they were larger than $2.5 \mathrm{~mm}$, and 'medium' if between the two sizes. The collector vein diameter was determined at the parenchymal level. DVA collector vein at contrast-enhanced T1-weighted sequence are shown in Fig. 2.

\section{Statistical Analysis}

IBM SPSS Statistics v. 23.0 was used for statisti- 


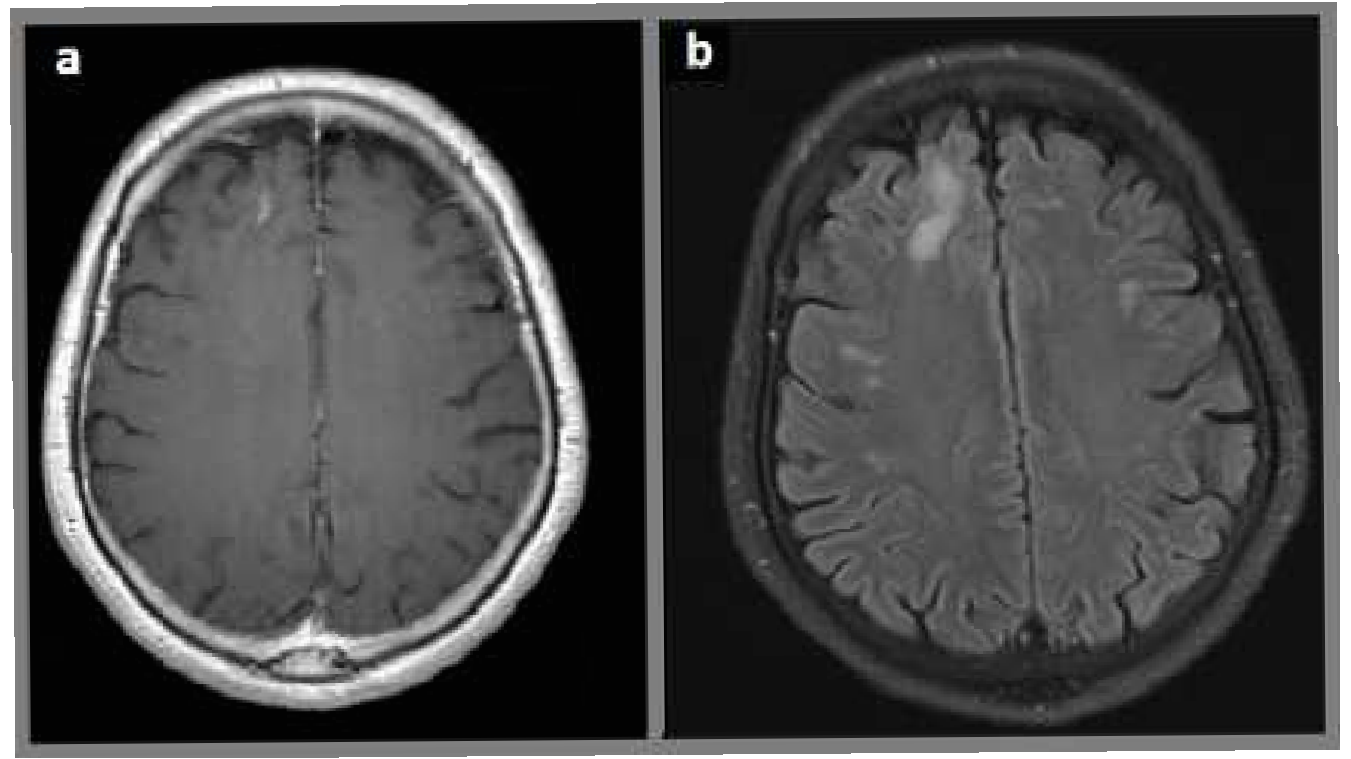

Fig. 2. (a) DVA collector vein at contrast-enhanced T1-weighted sequence, (b) DVA associated hyperintensity at FLAIR sequence.

cal analysis. For both groups, the statistical analysis was performed using chi-square and logistic regression methods for the defined characteristics of DVAs and age.

\section{RESULTS}

There were 84 patients in two groups. The age of the patients ranged from 12 to 80 , with the mean age being 38 years. The demographic findings and clinical presentations of the patients are given in Table 1, and their morphological findings in Table 2. There were

Table 1. Demographic characteristics and clinical presentations of the patients

\begin{tabular}{lc}
\hline Number of patients & 84 \\
\hline Female & 44 \\
Male & 40 \\
\hline Mean age & 38 \\
Headache & 38 \\
\hline Vertigo & 15 \\
\hline CVE & 12 \\
\hline Follow-up of mass & 9 \\
\hline Metastasis screening & 7 \\
\hline Epilepsy & 3 \\
\hline
\end{tabular}

$\mathrm{CVE}=$ cerebro vascular event
24 patients in the FLAIR (+) group 60 patients in the FLAIR (-) group.

When comparing the morphological variables in the chi-square test (Table 3 ), there was no statistically significant difference between the two groups in terms of DVA location and length ( $p>0.05)$; however, a statistically significant difference was found in terms of DVA width, and the logistic regression analysis revealed that medium and wide lesions had a higher probability of forming FLAIR hyperintensity compared to those that were thin. In terms of the DVA depth variable, no statistically significant difference was found between the FLAIR groups $(p=0.499)$; however, periventricular localization was observed in $33 \%$ of the patients in the FLAIR ( + ) group and $15 \%$ of those in the FLAIR (-) group.

In the evaluation of drainage localization, there was a statistically significant difference in the chisquare analysis of drainage to deep+superficial (D+Y) veins, but here it is necessary to note a statistical limitation resulting from the $\mathrm{D}+\mathrm{Y}$ observation value being zero. Consistent with this idea, the logistic regression analysis revealed that the drainage variable was not significant.

There was also a significant statistical difference in the age variable between the two groups, and the logistic regression analysis showed that the probability of DVA accompanied by FLAIR hyperintensity increased with age (Table 4). 
Table 2. Morphological findings of the groups

\begin{tabular}{|c|c|c|}
\hline & $\begin{array}{c}\text { FLAIR (-) } \\
n=60\end{array}$ & $\begin{array}{c}\text { FLAIR (+) } \\
n=\mathbf{2 4}\end{array}$ \\
\hline \multicolumn{3}{|l|}{ DVA location } \\
\hline Lobar & 40 & 21 \\
\hline Basal ganglion & 8 & 1 \\
\hline Cerebellum & 12 & 2 \\
\hline \multicolumn{3}{|l|}{ Length } \\
\hline Short & 17 & 5 \\
\hline Medium & 22 & 6 \\
\hline Long & 21 & 13 \\
\hline \multicolumn{3}{|l|}{ Width } \\
\hline Thin & 31 & 4 \\
\hline Medium & 21 & 16 \\
\hline Wide & 8 & 4 \\
\hline \multicolumn{3}{|l|}{ Drainage location } \\
\hline Superficial & 32 & 8 \\
\hline Deep & 28 & 13 \\
\hline Deep + Superficial & - & 3 \\
\hline \multicolumn{3}{|l|}{ Depth } \\
\hline Periventricular & 15 & 8 \\
\hline Juxtacortical & 16 & 7 \\
\hline Subcortical & 29 & 9 \\
\hline
\end{tabular}

DVA $=$ developmental venous anomaly

When the FLAIR classification of the variables was examined using logistic regression analysis, width and age were determined to be significant variables. Accordingly, as the age increased, the probability of being FLAIR $(+)$ increased. In addition, the patients with medium and wide DVAs were more likely to be in the FLAIR $(+)$ group compared to patients with thin DVAs (Table 5).

\section{DISCUSSION}

DVAs, similar to other venous structures of the brain, drain a parenchymal area. It is known that cavernoma, dystrophic calcification, and focal atrophy accompany DVAs at varying rates. Another finding observed in white matter areas that DVAs drain is
FLAIR and T2-weighted hyperintensity in this area that is prominent in the FLAIR sequence. In histopathological studies, demyelination in hyperintense areas, degenerative changes in nerve cells, and leukomalacia have also been described [12]. In the current study, we compared the hyperintensities in white matter with the morphological findings of DVAs and found that 26 of 189 patients (14\%) had hyperintensity in white matter. The rate of hyperintensity accompanying DVAs was reported as $12.5 \%$ by Santucci et al. [9] and $28.3 \%$ by Ruíz et al. [5]. These different rates may be due to the varying routine imaging protocols of the centers. In addition, the reason for the increased changes in white matter in the study of Ruíz et al. may be related to their higher rate of CT procedures [5].

No significant relationship was found between the 
Table 3. Chi-square analysis between FLAIR and investigated variables

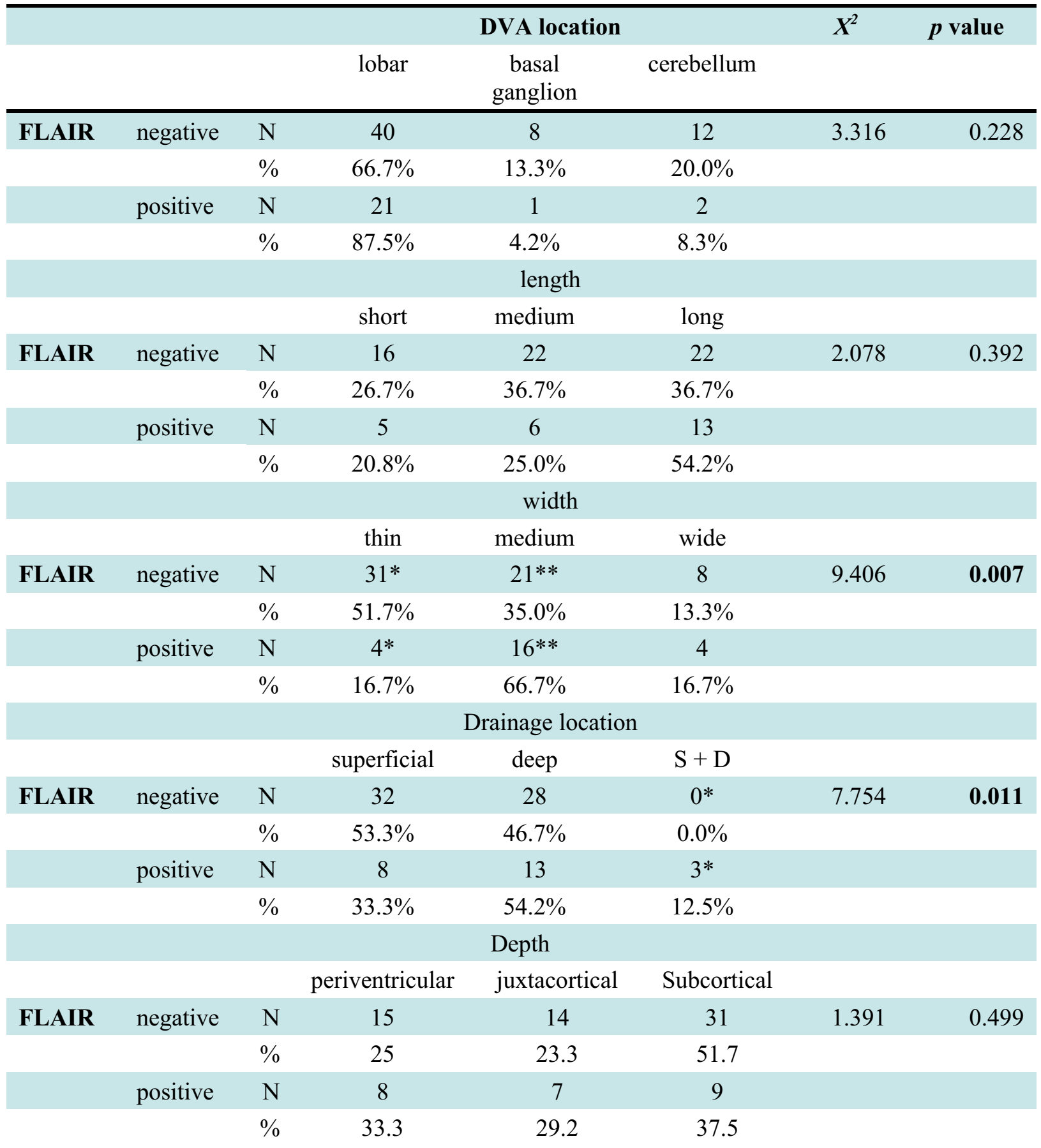

DVA $=$ developmental venous anomaly

Table 4. The relationship between FLAIR groups and age

\begin{tabular}{ccccccc}
\hline & FLAIR & N & Mean & Std. Deviation & t & $\boldsymbol{p}$ value \\
\hline \multirow{2}{*}{ Age } & Negative & 60 & 37.1667 & 16.10892 & -2.109 & 0.038 \\
& Positive & 24 & 45.3750 & 16.11862 & & \\
\hline
\end{tabular}


Table 5. Results of logistic regression analysis

\begin{tabular}{|c|c|c|c|c|c|c|c|c|}
\hline & \multirow[t]{2}{*}{ B } & \multirow[t]{2}{*}{ SE } & \multirow[t]{2}{*}{ Wald } & \multirow[t]{2}{*}{ Df } & \multirow[t]{2}{*}{ Significance } & \multirow[t]{2}{*}{$\operatorname{Exp}(B)$} & \multicolumn{2}{|c|}{$95 \%$ CI for $\operatorname{Exp}(B)$} \\
\hline & & & & & & & Lower & Upper \\
\hline Age & .051 & .020 & 6.642 & 1 & .010 & 1.052 & 1.012 & 1.094 \\
\hline Width (thin) & & & 8.484 & 2 & .014 & & & \\
\hline Width (medium) & 2.118 & .755 & 7.872 & 1 & .005 & 8.318 & 1.894 & 36.533 \\
\hline Width (wide) & 2.154 & .985 & 4.786 & 1 & .029 & 8.617 & 1.251 & 59.352 \\
\hline Constant & 3.121 & 7257.867 & .000 & 1 & 1.000 & 22.673 & & \\
\hline
\end{tabular}

$\mathrm{SE}=$ standard error, $\mathrm{CI}=$ confidence interval

presence of hyperintensity and DVA location, drainage system and length among the variables examined between the two groups, and this is consistent with the findings reported by Santucci et al. [9]. However, in contrast to Santucci et al. [9], we did not find a statistically significant relationship between the presence of hyperintensity and deep localization of DVAs. Santucci et al. [9] found periventricular localization at a higher rate in the DVA group accompanied by hyperintensity. Similarly, in our study, we observed periventricular localization to be more common in the FLAIR (-) group (33\%) than in the FLAIR (+) group (15\%), albeit non-significant. The depth classification of DVAs is made according to the localization of multiple small intramedullary veins draining into the collector vein, which may be difficult to distinguish, especially if the intramedullary veins are long and dense. This difficulty in evaluation may be the cause of the inconsistency between our findings and those of Santucci et al. [9].

Considering the system into which the collector vein is drained, no relationship was found between superficial or deep system drainage and hyperintensity, but it is interesting that in the sample of 84 patients, all three that showed $\mathrm{D}+\mathrm{Y}$ drainage were in the FLAIR $(+)$ group. Since we were not able to perform a statistical analysis due to the limited number of these patients, there is a need for further evaluations of DVAs exhibiting deep and superficial drainage to clarify this issue.

With increasing age, FLAIR hyperintensity occurs due to chronic small vessel disease in white matter. These changes in white matter are graded by the Fazekas classification according to their extent. While FLAIR hyperintensities are in the form of discrete punctate foci in Fazekas grade 1, grades 2 and 3 show diffuse and confluent tendencies. Grade 2 and 3 changes are common and were from the current study since they cannot be distinguished from DVA-induced hyperintensity. Thus, age-related white matter changes were excluded, allowing for a more accurate evaluation of the relationship between age and DVA hyperintensity.

Our patients had heterogeneous clinical presentation and primary pathology. It may be the limitation of the study if primary pathology effect the white matter. Because of this we exclude patient that have extensive white matter hyperintensity anywhere of the brain and cerebellum.

The collector vein diameter of DVAs differed between the FLAIR (+) and (-) groups. In the evaluation of the collector vein diameter, Santucci et al. [9] did not find any significant difference. [9] However, while Santucci et al. performed the collector vein diameter evaluation using numerical values for all veins, we divided the veins into three according to their diameters, as we previously described. We also performed the measurements from the SWI or contrast-enhanced T1-weighted sequence, in which the diameter of the collector vein can be seen more clearly. The results of our statistical analysis, show that the probability of accompanying hyperintense increased in DVAs with medium and wide collector veins compared to those with thin veins. This supports the hypothesis that chronic venous congestion plays a role in the formation of hyperintensity in white matter. In our opinion, the greater occurrence of chronic venous congestion in the presence of large-scale drainage veins may be the cause of detectable FLAIR hyperintensity in white matter. Venous congestion is also considered in the etiology of other pathologies of white matter drained by DVAs. For example, DVAs 
are thought to induce vascular proliferation by leading to hemodynamic instability in drainage localizations, thus leading to cavernoma [4], a theory that has been supported by DVA de-nova cavernoma cases reported in drainage localization $[13,14]$. If venous congestion occurs more in large collector veins, the collector veins of DVAs accompanying cavernoma may be wider. Further studies are needed to confirm this idea.

DVAs can also cause symptoms without concomitant cavernoma, albeit rarely. Symptomatic DVAs were classified by Pereira et al. [15] as mechanical due to the compression effect of the of the collector vein, increased inflow, decreased outflow or idiopathic. However, it remains unclear whether DVAs are more symptomatic in the presence of concomitant hyperintensity. In our study group, the rate of comorbidities was high; thus, we could not evaluate the relationship between the presence of hyperintensity and symptom formation.

In their study on FLAIR hyperintensities around DVAs, Rogars et al. [16] showed that concomitant demyelinating disease increased the rate of accompanying hyperintensity around DVAs. Histopathological studies also reveal demyelination in FLAIR hyperintense areas around DVAs. Concomitant demyelinating disease may increase the rate of demyelination in drainage localization. This hypothesis was supported by congestion shown in perfusion studies, but the number of perfusion cases in the literature is limited $[17,18]$.

\section{Limitations}

Our study has certain limitations. First, this study aimed to obtain information concerning the pathophysiology of white matter intensities that accompanied DVAs, but SWI scans were not available for all patients. The SWI sequence, now also known as SWI venography, has superior success in showing venous structures. In addition, considering that there may be cavernoma and micro bleeding accompanying DVAs, SWI and similar sequences are ideal for the diagnosis of these entities. The second limitation concerns the inability to eliminate cavernomas considering that the focus of the study was to investigate the etiology of white matter. In the literature, the most comprehensive studies on this subject belong to Ruíz et al. [5] and Santucci et al. [9]. In the study of Ruiz et al. [5], MRI was performed in 60 of 122 patients, but the scans did not include the SWI or gradient sequence. In the study of Santucci et al. [9], only three of the MRI examinations among 125 patients included the gradient sequence. In the current study, 17 of 84 patients did not have SWI examinations, and DVAs were diagnosed based on contrast-enhanced T1-weighted images. Two of these 17 patients were in the FLAIR (+) group. Further studies with available SWI sequences in all patients and a higher number of cases in all groups can provide more useful results in the etiology of hyperintensity accompanying DVAs.

\section{CONCLUSION}

In this study, we evaluated the changes in white matter in the drainage areas of DVAs and found a relationship between the collector vein diameter and patient age, supporting the association between hyperintensity formation and venous congestion. The findings suggest that there is a correlation between the degree and duration of venous congestion and the changes in white matter. Considering the results of our study and the literature, DVAs that are considered as benign and asymptomatic may actually form the basis of some pathologies. The issues of changes in white matter in relation to clinical presentation and cavernoma formation are still controversial and require more comprehensive longitudinal studies.

\section{Authors' Contribution}

Study Conception: ANŞÖ, BH; Study Design: ANŞÖ, BH, ÖÜ; Supervision: ANŞÖ, BH, ÖÜ; Funding: ANŞÖ; Materials: ANŞÖ; Data Collection and/or Processing: ANŞÖ, BH, ÖÜ; Statistical Analysis and/or Data Interpretation: ANŞÖ, BH, ÖÜ; Literature Review: ANŞÖ, BH, ÖÜ; Manuscript Preparation: ANŞÖ, BH, ÖÜ and Critical Review: ANŞÖ, BH, ÖÜ.

\section{Conflict of interest}

The authors disclosed no conflict of interest during the preparation or publication of this manuscript.

\section{Financing}

The authors disclosed that they did not receive any grant during conduction or writing of this study. 


\section{REFERENCES}

1. Ruíz DS, Yilmaz H, Gailloud P. Cerebral developmental venous anomalies: current concepts. Ann Neurol 2009;66:27183.

2. Lasjaunias P, Burrows P, Planet C. Developmental venous anomalies (DVA): the so-called venous angioma. Neurosurg Rev 1986;9:233-42.

3. Saito Y, Kobayashi N. Cerebral venous angiomas: clinical evaluation and possible etiology. Radiology 1981;139:87-94. 4. Wilson CB. Cryptic vascular malformations. Clin Neurosurg 1992;38:49-84.

5. Ruiz DS, Delavelle J, Yilmaz H, Gailloud P, Piovan E, Bertramello A, et al. Parenchymal abnormalities associated with developmental venous anomalies. Neuroradiology 2007;49:98795.

6. Huber G, Henkes H, Hermes M, Felber S, Terstegge K, Piepgras U. Regional association of developmental venous anomalies with angiographically occult vascular malformations. Eur Radiol 1996;6:30-7.

7. Ostertun B, Solymosi L. Magnetic resonance angiography of cerebral developmental venous anomalies: its role in differential diagnosis. Neuroradiology 1993;35:97-104.

8. Ge Y, Zohrabian VM, Osa EO, Xu J, Jaggi H, Herbert J, et al. Diminished visibility of cerebral venous vasculature in multiple sclerosis by susceptibility-weighted imaging at 3.0 T J Magn Reson Imaging 2009;29:1190-4.

9. Santucci GM, Leach JL, Ying J, Leach SD, Tomsick TA. Brain parenchymal signal abnormalities associated with developmental venous anomalies: detailed MR imaging assessment. Am J Neu roradiol 2008;29:1317-23.

10. Aoki R, Srivatanakul K. Developmental venous anomaly: benign or not benign. Neurol Med Chir 2016;56:534-43.

11. Lee C, Pennington MA, Kenney CM 3rd. MR evaluation of DVAs: medullary venous anatomy of venous angiomas. AJNR Am J Neuroradiol 1996; 17:61-70.

12. Noran HH. Intracranial vascular tumors and malformations. Arch Pathol 1945;39:403-4.

13. Maeder P, Gudinchet F, Meuli R, de Tribolet N. Development of a cavernous malformation of the brain. Am J Neuroradiol 1998;19:1141-3.

14. Campeau N, Lane J. De novo development of a lesion with the appearance of a cavernous malformation adjacent to an existing developmental venous anomaly. Am J Neuroradiol 2005;26:156-9.

15. Pereira VM, Geibprasert S, Krings T, Aurboonyawat T, Ozanne A, Toulgoat F, et al. Pathomechanisms of symptomatic developmental venous anomalies. Stroke 2008;39:3201-15.

16. Rogers DM, Shah LM, Wiggins RH. The central vein: FLAIR signal abnormalities associated with developmental venous anomalies in patients with multiple sclerosis. AJNR Am J Neuroradiol 2018;39:2007-13.

17. Camacho DL, Smith JK, Grimme JD, Keyserling HF, Castillo M. Atypical MR imaging perfusion in developmental venous anomalies. Am J Neuroradiol 2004;25:1549-52.

18. Kroll H, Soares BP, Saloner D, Dillon WP, Wintermark M. Perfusion-CT of developmental venous anomalies: typical and atypical hemodynamic patterns. J Neuroradiol 2010;37:239-42. 